\title{
Decision Analysis Using the Benefit-Cost-Deficit model
}

\author{
Karima SEDKI, ${ }^{1}$ Philippe Polet, ${ }^{2}$ Frédéric Vanderhaegen ${ }^{3}$ \\ ${ }^{1,2,3}$ Université Lille Nord de France, F-59000 Lille, France \\ UVHC, LAMIH FRE CNRS 3304, F-59313 Valenciennes, France
}

\begin{abstract}
In this paper, we are interested in the decision analysis focusing on the Benefit-Cost-Deficit $(B C D)$ model. The $B C D$ model is proposed recently in order to analyse the intentional deviated users behaviour (prescribed behaviour crossing). This later is considered as a decision problem under risk where the consequences of users' decisions can be evaluated following three attributes: the Benefit, the Cost and the Deficit. This paper proposes to consider the context of the decision, so we extend the $B C D$ model by introducing different organizational and human factors that influence the users in their actions. This means that the deviation of users behaviour is not only related to their voluntary and intention but also to other factors, such as those of the organization of the system that has also some objectives to achieve as improving production and quality. To build the decision model based on the $B C D$ approach, we propose a graphical representation using influence diagrams. The advantage of the proposed framework is to analyse the users' decisions in terms of benefit or risk considering the context (as the organisational factors).
\end{abstract}

Keywords: Decision problem, BCD model, risk, Influence diagram,

\section{Introduction}

Human users play the major role in the development of a system. Their actions can be considered as a potential source of performance and safety, but also as a source of errors because of neglecting the prescribed rules. Users can therefore act in the system with intentions, goals, with their skills and intelligence, pressure, fatigue, lack of motivation. All these factors can cause a considerable risk and many failures in the system. To protect or prevent the system ${ }^{1}$ from human errors and other undesirables consequences of their decisions, rules concerning the prescribed behaviour, material or symbolic means are placed. For example, in the restaurant, to prepare a good omelette, it is prescribed to firstly break the eggs in a cup to verify if they are good or rotten. However, the agent (or user) can cross this prescribed behaviour in order

\footnotetext{
${ }^{1}$ Following the studied problem, the system can be industrial, socio-technical, university, restaurant, hospital, etc.
}

to save a time or avoid to wash a cup for example. So, if the egg is rotten, there is risk of wasting an omelette and dissatisfaction of clients. It is the same thing on roads where the drivers often try to cross the highway code: overtake the continuous line or the red light for example. As these two problems, in different real problems, the decision making can be considered in different form: under risk, certainty and uncertainty $[5,6,4,14]$. For the authors in [4], the decision is under certainty when each action is known to lead in a specific consequence, under risk when each action deals to one of a set of possible consequences, each consequence occurring with a known probability and under uncertainty when actions may lead to a set of consequences without known probabilities.

In [7], the authors have developed a model called $B C D$ (Benefit, Cost, Deficit) that allows to study and explain the deviation of the prescribed behaviour by users. The user crossed the prescribed behaviour (or neglect the prescribed rules) in order to achieve some personal interests that are related for example to workload, productivity. Namely, this model is based on the idea that the decision of crossing a prescribed behaviour may be beneficial for the users and the system. But since the objective of any prescribed behaviour is to protect the system and the users, the BCD model allows also to evaluate the possible negative consequences of user's decisions, namely the costs and the potential deficit are also introduced.

The deviation of the prescribed behaviour by users can be considered as a decision problem under risk. If the user respects the prescribed behaviour, then probably, the decision has no risk in the system, otherwise, the consequences can be negatives (potentials deficits) or positives (benefits). In this paper, we propose a framework that allows to represent and analyse such decision problem in the presence of uncertainty and multiple criteria. We consider that user's decisions are also influenced by some organisational (as production pressures) and human factors (as lack of experience). One of the main aims of a decision analysis in terms of benefit and risk is to evaluate and/or validate a described behaviour concerning a given system. Regarding analysis' results, some new rules may be added or strictly prohibited in order to improve the safety 
level, the quality, etc. But the addition of new rules or prohibition of some rules may lead to difficulties to achieve productivity goals for example. So, it is important to consider a multi-criteria approach for an efficient analysis.

Thus, to model such a decision problem, we propose to use an influence diagram that has the advantage to handle uncertainty, represent interdependencies between the different decision variables (organisational and human factors, decisions, criteria and their importance) and quantify the utility of each decision regarding the benefit, cost and deficit.

The paper is organised as follows: in section 2, we present the $B C D$ model, Section 3 presents our proposed model. Section 4 gives an illustrative example and the final section concludes the paper.

\section{The BCD model}

The authors in [7] describe an intentional deviated behaviour from human users, where consequences can be analysed following three parameters:

- The benefit (B): it represents the gains associated (searched by the user) with crossing a prescribed behaviour.

- The cost (C) : corresponds to the acceptable losses for the user in order to achieve the expected benefits.

- The deficit (D) : represents unacceptable losses due to possible failures of crossing the prescribed behaviour.

The crossing of the prescribed behaviour from users is considered as a particular case of decision making under risk. An action $A$, when is executed, can give two types of situations (consequences):

- Success: it concerns the situation where the execution is correct, the consequence will be noted $C S(A)$. It corresponds to the desired consequence for the user.

- Failure: it concerns the case where the execution is incorrect. The consequence (noted $C F(A)$ ) of an action does not correspond to the desired result.

When a user decide to respect or cross a prescribed behaviour, two actions are to be evaluated:

- The prescribed action which corresponds to the respect of the prescribed behaviour. This action is noted $P$.

- The obtained action of crossing the prescribed behaviour. It is noted $D$.

To determine the benefits, costs and deficits, the authors in [7] propose a comparison between the consequences of the prescribed behaviour and the deviated one. The comparison is done as follows:
$B_{i}(F B) \quad\left(\right.$ rep. $\left.\quad C_{i}(D), D_{i}(D)\right)$ represents the benefits (resp. the costs and the deficits) related to the deviation of the prescribed behaviour for each criterion $i$ :

$$
\begin{aligned}
& \text { if }\left(C S_{i}(D)-C S_{i}(P)\right)>0 \\
& \text { then } \\
& B_{i}(D)=C S_{i}(D)-C S_{i}(P) \text { and } C_{i}(D)=0 \\
& \text { else } \\
& C_{i}(D)=C S_{i}(P)-C S_{i}(D) \text { and } B_{i}(D)=0 \\
& \text { if }\left(C F_{i}(D)-C F_{i}(P)\right)>0 \\
& \text { then } \\
& D_{i}(D)=C F_{i}(D)-C F_{i}(P) \\
& \text { else } \\
& D_{i}(D)=0 .
\end{aligned}
$$

\section{Proposed model}

We propose a model that allows to represent the treated problem and analyse each decision regarding the context (factors, consequences) using influence diagram (ID). This model extends the $B C D$ model by introducing organisational and human factors that may influence the users in their actions. Such factors can be defined following the studied problem and the objectives of the concerned system. Our motivation to use ID for solving the treated problem can be illustrated by two main reasons: the first one is that any decision problem requires the modelling step, namely the identification of the decision components and relationships between them. Indeed, using ID which is simultaneously a graphical and mathematical model allows to represent all the required elements in the same model in an expressive and clear way. In addition, uncertainty and interdependencies between the elements are easily represented. The second reason is the possibility to order the set of decisions by simply applying the inference in the constructed model (we note that in order to compute the utility of each alternative, we should define a utility function in the model).

\subsection{Organisational factors}

The objective of considering organisational factors in decision under risk is to identify the factors of the organisation may impact the users' actions and occurring in an negatives consequences. These factors can concern the production, the safety, control, etc. In [11], seven organisational factors are defined in order to study the risk analysis of industrial systems, they are based on [9] depicting the organization in a global way and describing it by pathogenic organizational factors: Shortcomings in the Organization Culture of Safety, Failure in Daily Safety Management, Weakness of Control, poor handling of Organizational Complexity, etc. Such factors contribute to affect principally the safety and to cause or precipitate the accident. Considering the context in decision making by examining the culture 
of the organisation is important since this affects the way the decision is made $[12,13]$.

\subsection{Human factors}

Each user action (or decision) is characterized by items that impact its effectiveness. These items can concern: experience, formation, respect or not the work specifications, feedback experience, etc. When a given organisation factor represents a pathogenic view, this can impact the users' decisions (for example, when production pressure are presented, can cause stress and lack of concentration of users). Human factors play an important role in users' decisions. As cited in [10], statisticals of Transportation Safety Board of Canada show that $74 \%$ of accidents at sea are dues to human errors (as errors dues to comprehension between the pilot and the captain, lack of communications, etc.).

We present in the following a briefer description of influence diagrams that we use to built our model.

\subsection{Influence diagrams (ID)}

ID are a directed graphical models for decision making under uncertainty. They extend Bayesian networks and structured on three types of variables: decision, chance (or uncertain) and utility nodes (see example of Figure 1). Decision nodes correspond to variables that are under the control of the decision maker, chance and utility nodes correspond to variables that are not controlled by the user. They respectively represent beliefs and preferences. Arcs directed from chance nodes toward decision nodes indicate that the probability distribution of these chance variables are affected by the decision (i.e these chance variables will be observed before the decision is made). Arcs towards chance nodes indicate that the probability distribution of these variables are conditional on their parents (Conditional Probability Table (CPT)). Like chance nodes, a utility node is represented by a table of utility values in the context of its parents nodes.

Figure 1 presents an ID about the decision problem of taking an umbrella [2]. It contains two chances nodes (Forecast and Weather) containing probabilistic information about the forecast and the weather. Decide Umbrella is a decision node with two outcomes (take it, leave it at home). Satisfaction is a utility node which represents the values of desirability of each decision regarding the weather. The objective of this example is to compute an expected utility for each decision (take it, leave it at home) and return the one with the highest utility.

\subsection{The structure of the proposed model}

The structure of our model contains different variables that are defined in the following:

- Variables concerning the organisational factors that are described previously.

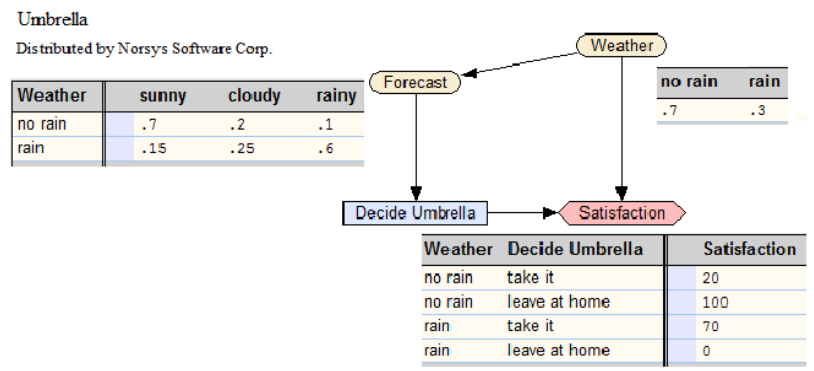

Figure 1: An example of influence diagram.

- Human factors that correspond to the items characterizing the human actions.

- Variables that allow to define the prescribed and deviated behaviour. For example, the required time for executing an action, the number of necessary steps, etc.

- Variables that represent different criteria (example : quality, productivity, workload, etc.). We consider that each criterion is associated with a weight which represents the importance of this criterion for the organisation.

- Variables that represent the consequences of the deviated behaviour: benefit, deficit, cost, optimistic utility, pessimistic utility and final utility which represents the risk of each action.

Figure 2 illustrates the general schema of the proposed model.

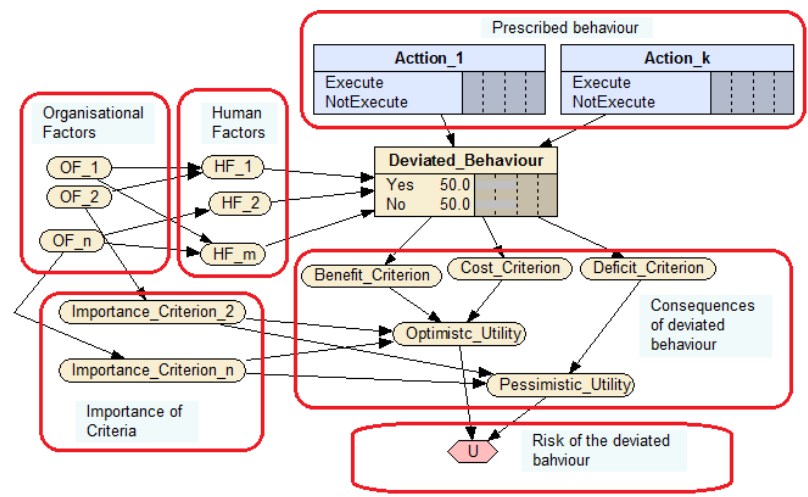

Figure 2: The proposed model.

\subsection{Model parameters}

The variables representing the organisational factors (OF), human factors (HF), importances of criteria, and some variables that are related to the consequences of decisions (benefit, cost, deficit, optimistic utility, pessimistic utility) are represented as chance nodes in the graph. The users' actions corresponding to the prescribed behaviour are represented as decision nodes with two possible states (Execute (Ex), NotExecute (NEx)). The utility node $(U)$ allows to quantify the utility value of each decision. Each OF is associated with two possi- 
bles states (present, absent). When a given organisational factor is present, this means that the pathogenic view of this factor is present (for example, the FO production pressure is present, means that there are pressures of production in the organisation, such factor impact the users in their actions). It is also the same case for each human factor (present, degraded). For example, the formation of users can be degraded. The importance of each criterion (symbolised by $I m p_{i}$ ) is evaluated in the unit $[0,1]$ interval (see Table 1 ).

\begin{tabular}{|c|c|}
\hline Evaluation & Criterion Importance $\left(\operatorname{Imp} p_{i}\right)$ \\
\hline \hline $0-0.2$ & not important \\
$0.2-0.6$ & medium \\
$0.6-1$ & important \\
\hline \hline
\end{tabular}

Table 1: Values associated with criterion importance.

Different variables are defined to evaluate each decision, they are defined as follows:

Benefit, Cost and Deficit: these variables are defined in section 2 ( $B C D$ model).

Pessimistic Utility $(U p)$ : this variable represents the utility of the worst consequence, i.e it is defined following the obtained deficit when the decision is taken. $U p$ is computed as follows:

$U p=\sum_{i=1, \ldots, n}\left(\operatorname{Imp}_{i} * D_{i}\right) / \sum_{i=1, \ldots, n} \operatorname{Imp}_{i}$ where $I m p_{i}$ is the value of importance associated with each criterion $i, n$ is the set of criteria and $D_{i}$ are the obtained deficit for each criterion.

Optimistic Utility $(U o p)$ : this variable represents the utility of the best consequence, i.e it is defined following the obtained benefit and cost. $U o p$ is defined as follows :

$U o p=\sum_{i=1, \ldots, n}\left(\operatorname{Imp}_{i} *\left|B_{i}-C_{i}\right|\right) / \sum_{i=1, \ldots, n} \operatorname{Imp}_{i}$

where $I m p_{i}$ is the value of importance associated with each criterion $i, n$ is the set of criteria, $B_{i}, C_{i}$ are the benefit and cost obtained for each criterion $i$.

The optimistic and pessimistic utilities are evaluated in the unit $[0,1]$ interval (see Table 2).

Final Utility $(U)$ : this variable represents the risk or the benefit of the taken decision. The value of $U$ is negative, when the pessimistic utility is greater than the optimistic utility. In this case, the decision is risky. Otherwise, the taken decision brings benefits. $U$ is computed as follows: $U=$ $U o p-U p$

\section{Illustrative Example}

Let us study in this section the Omelette example introduced by Savage [3]. In this example, an agent (or a user) has a cup, an omelette of 5 eggs and another egg that he should decide whether or not to

\begin{tabular}{|c|c|c|}
\hline Evaluation & Optimistic Utility & Pessimistic Utility \\
\hline \hline $0-0.2$ & very low & very low \\
$0.2-0.4$ & low & low \\
$0.4-0.6$ & medium & medium \\
$0.6-0.8$ & high & high \\
$0.8-1$ & very high & very high \\
\hline \hline
\end{tabular}

Table 2: Values associated with optimistic and pessimistic Utilities

add it to a 5 eggs omelette knowing that the available egg may be rotten. Assume that the problem concerns a restaurant which has many objectives: satisfy the clients ensuring less waiting time, obtain a good omelettes and not using more than laid number of eggs. So, as there is uncertainty about the state of the egg, the prescribed behaviour in this restaurant is that each agent should break the egg in a cup (action bo) in order to verify if it is good or rotten. Break the egg directly on the omelette (bc) or throw it (te) away can be considered as a deviated behaviour of the user. The organisational factors concerning this problem can correspond to: Weakness of Control, Production Pressures. The criteria can concern the production (good omelette) and waiting time. The human factors can be Respect of work specification which can be present or degraded. The possible consequences of the actions are represented in the following table:

\begin{tabular}{|l|c|c|}
\cline { 2 - 3 } \multicolumn{1}{c|}{} & \multicolumn{2}{c|}{ State of the egg } \\
\hline Actions $\left(A_{i}\right)$ & the egg is good & the egg is rotten \\
\hline bo & $\begin{array}{c}6 \text { eggs omelette and } \\
\text { cup to wash }(3)\end{array}$ & $\begin{array}{c}5 \text { eggs omelette and } \\
\text { cup to wash }(4)\end{array}$ \\
\hline bc & 6 eggs omelette (1) & wasted omelette (2) \\
\hline te & $\begin{array}{c}5 \text { eggs omelette } \\
(1 \text { wasted egg) }(5)\end{array}$ & 5 eggs omelette $(6)$ \\
\hline
\end{tabular}

Table 3: Example representing the omelette problem using BCD model

Table 4 gives the consequences of each decision in terms of benefit, cost and deficit.

\begin{tabular}{|c|c|c|}
\hline & \multicolumn{2}{|c|}{ State of the egg } \\
\hline$A_{i}$ & the egg is good (CS) & the egg is rotten $(\mathrm{CF})$ \\
\hline bo & $\begin{array}{c}C S \_ \text {Prodution }=6 \text { eggs } \\
C S \_ \text {Time }=10 \mathrm{mn}\end{array}$ & $C F \_$Production $=5$ eggs \\
\hline $\mathrm{bc}$ & $\begin{array}{c}C S \_ \text {Prodution }=6 \text { eggs } \\
\text { CS_Time }=8 \mathrm{mn} \\
\text { (Benefit Time) }\end{array}$ & $\begin{array}{c}C F_{-} \text {Prodution }=-5 \text { eggs } \\
\text { (Deficit production) }\end{array}$ \\
\hline te & $\begin{array}{c}C S_{\text {_Prodution }=5 \text { eggs }} \\
\text { (Cost production) } \\
C S_{\text {_Temps }}=8 \mathrm{mn} \\
\text { (Benefit Time) }\end{array}$ & $C F \_$Prodution $=5$ eggs \\
\hline
\end{tabular}

Table 4: Example representing the omelette problem (consequences using BCD model) 
Concerning this problem, we consider that when the actions of the prescribed behaviour are executed with success, then the consequences regarding the production criterion are 6 eggs omelette $\left(C S \_\right.$Prodution $=6$ eggs $)$, and the required time to make the omelette is 10 minutes (CS_Time $=10 \mathrm{mn})$. When the prescribed behaviour fails (case where the egg is rotten for example), then we have $C F \_$Prodution $=5$ eggs and $C F \_$Time $=10 \mathrm{mn}$ (we suppose that we do not remake the omelette). This means that concerning the prescribed behaviour, we have 0 benefit, 0 cost and 0 deficit. Concerning the deviated behaviour 1 (action bc), when the egg is good, there is a benefit in terms of time (2 minutes). However, when the egg is rotten, there is deficit (wasted omelette, deficit $=-5$ eggs). Concerning the deviated behaviour 2 (action te), when the egg is good, the consequences are : cost for production criterion (1 good egg is wasted) and benefit for the time criterion (= 2 minutes) because of throwing the egg. This means that the deviated actions are beneficial if their positives consequences are more important than those of the prescribed behaviour (i.e, benefit if the production concerns more than 6 eggs, and the time is least than 10 minutes), and risked (deficit) if their negatives consequences are worst than those of the prescribed behaviour in case of failure (deficit if the production concerns less than 5 eggs, and the time more than 10 minutes).

Figure 4 represents the influence diagram which models the presented problem. The models contains the following variables:

- Two organisational factors (OF) (Production_Pressure and Control) with two possible states for each one (Present, Absent). Probabilistic informations about these two variables can be given as observations: for example (Production_Pressure is Present with probability 0.7, Absent with probability 0.3). The factor Control can influence the users' actions in terms of quality and time for example.

Two human factors (Respect_Of_Work_Specifications and Stress), with two states for each one (Yes, No).

- Two criteria (Production and Time). Each decision is evaluated regarding each criterion in terms of Benefit, Cost, Deficit. And, each criterion is associated with a weight (importance criterion) as defined in Table 1 . Figure 3 shows the value of importance concerning the production criterion (Imp_Prodution).

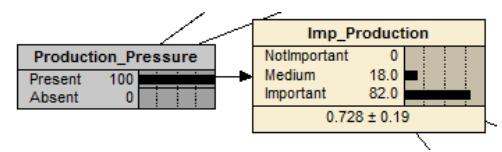

Figure 3: Importance of Production criterion.

- Egg_State: This variable presents probabilistic

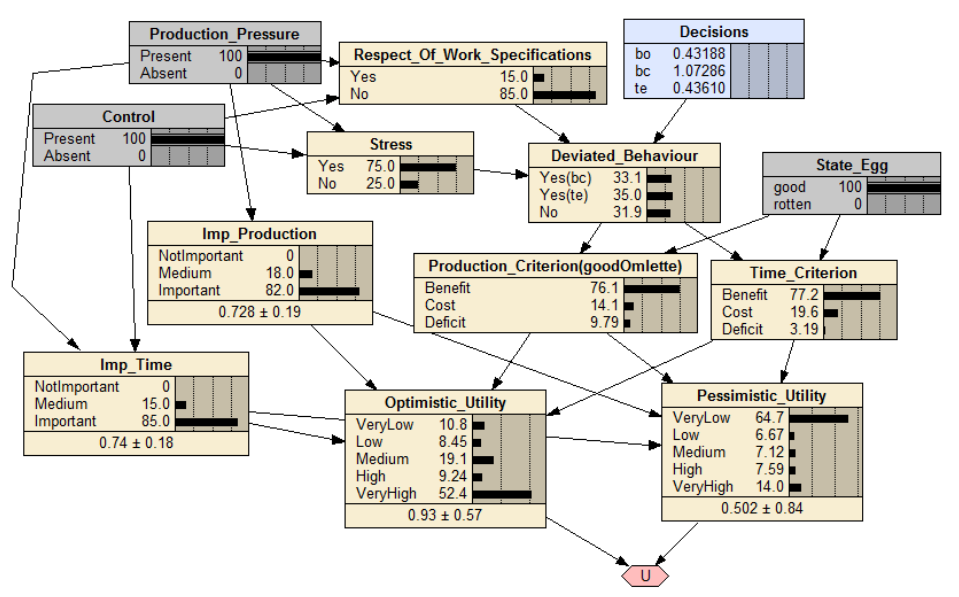

Figure 4: Results concerning case 1.

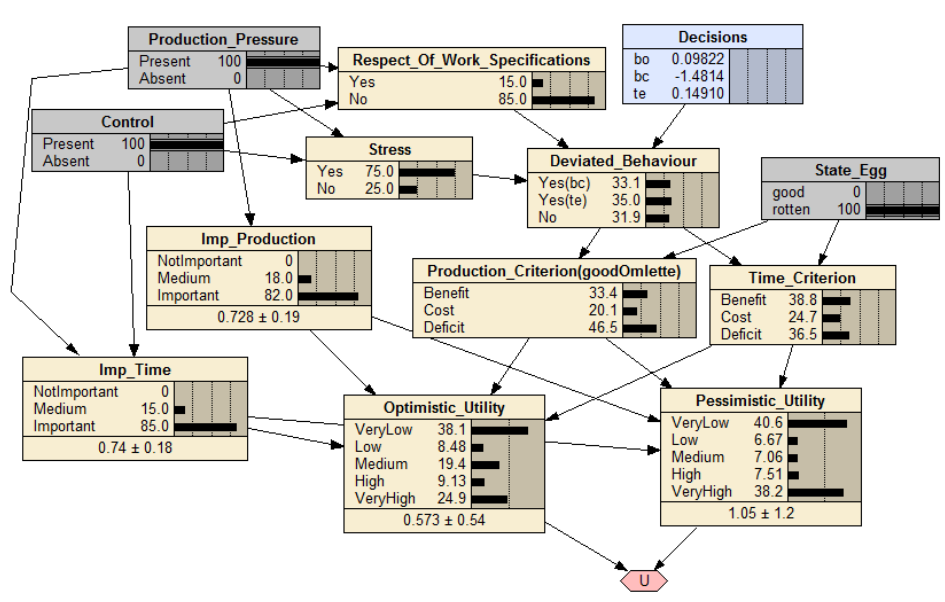

Figure 5: Results concerning case 2.

information about the state of the additional egg. - Optimistic_Utility: It is defined in the aim to directly observe the positive consequences of a given decision. Pessimistic_Utility is also given to observe the negative consequences of a given decision. We note that these two nodes can be deleted in the ID without influence on the final utility (but they allow to better understand the situation of each decision).

- The unique decision node contains 3 possible actions. In this problem, as there is only one prescribed action, we consider that the user can execute one of the three actions $(b c, b o$, te which are mutually exclusive).

- The Utility node allows to compute the utility of each decision regarding the observations about the factors and other variables in the graph. The value of each decision is displayed in the node decision (see Figure 4).

\subsection{Illustrative cases}

1. Case 1 (see Figure 4): The observations are: State_Egg = good, Production_Pressure $=$ 
Present, Control = Present. The obtained results are: Imp_Time $=0.74$ (Important), Imp_Production $=0.728$ (Important). The decisions are ranked as follows: $b c(U=$ $1.07286)$, $t e(U=0.43610)$, bo $(U=0.43188)$. The action $b c$ is the best one (in this case, the user obtains a good 6 omelette without a cup to wash). The decision te is somewhat better than bo because the Time criterion is lightly higher than Production (namely, ensuring the less waiting time for the clients is more important than produce the maximum omelette for the restaurant in this case).

2. Case 2 (see Figure 5): The observations concerning $\mathrm{OF}$ and $\mathrm{HF}$ are the same in case 1, but State_Egg = rotten. We observe that the decision $b c$ is the most risky (negative utility).

\section{Conclusion}

In this paper, we presented an approach for decision analysis focusing on a Benefit-Cost-Deficit $(B C D)$ model. The model allows to analyse and evaluate the consequences of the intentional deviated human behaviour. To build the model based on the $B C D$ approach, we proposed a graphical representation using an influence diagram. We proposed to take into account the context of the decision by introducing different factors that influence the users in their actions (as organizational and human factors). Including such factors is important, namely for evaluation the consequences (negative or positive). In addition, the fact that the analysis of the decision is done in terms of Benefit, Cost and Deficit, this allows to improve the prescribed behaviour in terms of means, rules and informing the users about the failures that some decisions can cause in the system (namely, when the safety is the important one). So, such approach is important, namely for risk analysis. Concerning future work, it may be interesting to get another evaluation of Benefit, cost and deficit in terms of fuzzy set approach and possibility theory, namely in order to represent the ignorance about the consequences of the deviated behaviour. We are also interested to apply this approach on an example of the use of an industrial rotary press. In this problem, deviations between the prescribed operational use and the operational use from users are possible. These deviations can affect the safety, quality or production objectives of the machine. The rotary press can pose many hazardous conditions for the users, such as burns, cuts or falls, excessive noise, intoxication. Moreover, production losses are rapidly increasing when the rotary press is not operating well. Different incidents can disturb the production: breaking of the printing plate, over-tightening of the screw that provides ink, etc.

\section{Acknowledgement}

This work is supported by the SOMAIR project funded by the group of Scientific Interest "Monitoring, Security and Safety Systems Department (GIS 3SG).

\section{References}

[1] J. Wiley, editor. Influence Diagrams, Belief Nets and Decision Analysis, R. M. Oliver and J. Q. Smith, 1999.

[2] UCL Press, editors. An Introduction to Bayesian Networks, Jensen, Finn V. 1996.

[3] John Wiley \& Sons editor. The Foundations of Statistics, Leonard Jimmie Savage, New York 1954.

[4] John Wiley \& Sons editor. Games and Decisions. R. D. Luce and H. Raiffa, 1957.

[5] Princeton University Press. Theory of games and economic behavior, von Neumann, J. and Morgenstern, O., Princeton, N.J., 1947.

[6] D. Guyonnet, B. Bourgine, D. Dubois, H. Fargier, Bernard CÃ'me, J.-P. Chil ̃̃ "s. Hybrid Approach for Addressing Uncertainty in Risk Assessments. Journal of Environmental Engineering 129(1): 68-78, 2003.

[7] Polet, P. and F. Vanderhaegen and P. Millot and P.A. Wieringa, Barriers and risk analysis. In Symposium on Analysis Design and Evaluation of Human Machine Systems, 2001.

[8] E. Hollnagel, Accident and barriers. In Proceedings of 7th European Conference on Cognitive Science Approaches to Process Control, pages 175-180, France, 1999.

[9] Pierlot, S., Y. Dien and M. Llory (2007). From organizational factors to an organizational diagnosis of the safety. ESREL, 2, 1329-1335, 2007.

[10] Trucco P., Di Giulio A., Randazzo G., Pedrali M. Towards a systematic organisational analysis for improving safety assessment of the maritime transport system, in safety and reliability, Bedford \& Van Gelder (eds), pp. 513-521, ESREL, 2003.

[11] Léger, A. and R. Farret and C. Duval and E. Levrat and P. Weber and B. Lung, A safety barriers-based approach for the risk analysis of socio-technical systems, in Seventeenth IFAC Word Congress, Séoul, Corée du Sud, 2008.

[12] J. March. (ed.) (1987), Handbook of organizations. New York, Garland Pub.

[13] Ari Riabacke. Managerial Decision Making Under Risk and Uncertainty.IAENG International Journal of Computer Science, 32:4, 2006.

[14] Hélène Fargier et Régis Sabbadin. Qualitative decision under uncertainty : back to expected utility. Journal of Artificial Intelligence, 164 :245-280, 2005. 